\title{
Magnetic resonance imaging protocols for paediatric neuroradiology
}

\author{
Dawn E. Saunders • Clare Thompson • \\ Roxanne Gunny $\cdot$ Rod Jones $\cdot$ Tim Cox • \\ Wui Khean Chong
}

Received: 6 February 2007 /Revised: 2 March 2007 / Accepted: 14 March 2007 / Published online: 9 May 2007

(C) Springer-Verlag 2007

\begin{abstract}
Increasingly, radiologists are encouraged to have protocols for all imaging studies and to include imaging guidelines in care pathways set up by the referring clinicians. This is particularly advantageous in MRI where magnet time is limited and a radiologist's review of each patient's images often results in additional sequences and longer scanning times without the advantage of improvement in diagnostic ability. The difficulties of imaging small children and the challenges presented to the radiologist as the brain develops are discussed. We present our protocols for imaging the brain and spine of children based on 20 years experience of paediatric neurological MRI. The protocols are adapted to suit children under the age of 2 years, small body parts and paediatric clinical scenarios.
\end{abstract}

Keywords MRI $\cdot$ Protocols $\cdot$ Children

\section{Introduction}

The use of imaging protocols allows the standardization of procedures and workflow as well as consistency of image quality. With appropriate training, radiographers can select and implement imaging protocols with relatively little requirement for radiological involvement. This use of skill mix provides radiographers with an expanded role and greater responsibility that increases job satisfaction and staff retention.

D. E. Saunders $(\bowtie) \cdot C$. Thompson $\cdot$ R. Gunny $\cdot$ R. Jones $\cdot$

T. Cox $\cdot$ W. K. Chong

Department of Radiology, Great Ormond Street Hospital,

Great Ormond Street,

London WC1N 3JH, UK

e-mail: SaundD@gosh.nhs.uk
Imaging protocols can also provide guidance for radiologists and radiographers for patients in shared care between secondary and tertiary care institutions. The use of protocols set up by the referring centre will prevent repeat MR scanning with the additional burden on MR scanner time. These advantages are offset by the minor disadvantage that radiologists' personal preferences and the intricacies of individual scanners are not taken into consideration.

At Great Ormond Street Hospital (GOSH), MRI protocols have been implemented for nearly 20 years and have been modified as scanners are replaced and new sequences developed. Our success is measured by the minimal number of patients recalled (less than 1\%). Increasingly, we are asked about our MR protocols by departments throughout the United Kingdom and have set them out here to make them freely available to those who may find them beneficial to patient care. The protocols provided are confined to conventional imaging techniques and diffusion-weighted imaging (DWI), and do not include the advanced imaging techniques of perfusion imaging, diffusion tensor imaging and MR spectroscopy that remain largely within the research domain.

\section{Practical issues of MRI in children}

One of the biggest challenges of paediatric neuroimaging is the acquisition of high-quality diagnostic images, as it requires the infant or child to keep still for a long period of time (sometimes nearly $1 \mathrm{~h}$ ). Children over the age of 7 years are scanned, where possible, without sedation and with recourse to play specialists and videos. Neonates under 2 months old (corrected age, if premature) are scanned during natural sleep induced by food, comfort and warmth (feed and wrap), often best after a period of sleep deprivation. If this fails then light oral sedation, e.g. chloral 
hydrate, may be used. The majority of our patients (approximately $70 \%$ ) require sedation or a general anaesthetic. When sedation is required, it is usually heavy and heavier than that required for $\mathrm{CT}$ or nuclear medicine studies. Sedation is applied by specially trained nurses who are trained to decide when and how much to administer and monitor its effects, and who are skilled in the recovery of the child [1]. Details can be found in a review of the management of painless imaging in children [2].

All MR sequences may disturb the sleeping infant or child and ear protection such as earplugs and baby earmuffs should be used. Some motion can be avoided by swaddling infants, keeping them warm, and by placing moulded foam or airbags around the baby's or child's head. Cardiorespiratory monitoring with MR-compatible equipment is essential in all infants, whether sedated or not, and all sedated or anaesthetized children [2].

Commercial MR machines are designed for adult practice and few manufacturers make provision for the issues encountered in paediatric practice. Currently, most children are imaged in units that have a wider adult practice with only a handful of institutions throughout the world having dedicated paediatric MR facilities. The structures we need to examine in children are generally small and we aim for a maximum slice thickness of $5 \mathrm{~mm}$ in the brain and $3 \mathrm{~mm}$ in the spine. The slice thickness is reduced to $3 \mathrm{~mm}$ when acquiring images of the pituitary gland and orbits.

We do not recommend ultrafast 'breath-hold'-type T2weighted (T2-W) sequences that have reduced contrast to noise as an alternative to sedating a child, as achieving high contrast to noise is of utmost importance given the small size of the paediatric head. Advances in coil design have improved signal-to-noise and we now use multichannel head and spine coils.

\section{Practical consideration of the changing brain with age}

Changes in normal values of measured parameters in very young children occur as the brain undergoes very rapid development during the first $2-3$ years of life. This necessitates the creative modification of pulse sequence parameters in order to optimize the soft-tissue contrast between anatomical and pathological structures. The two most important physiological changes to take place within the first 2-3 years are reduction in the total brain water content and $\mathrm{T} 1$ and $\mathrm{T} 2$ shortening largely as a consequence of the maturation of myelin [3].

From birth to 6 months of age the maturation of myelin is best assessed using T1-weighted (T1-W) images. From 6 months until the completion of myelination at around 2 years of age, T2-W images are more useful. During the process of myelination on T1-W images the signal from white matter goes from dark to bright. For a variable period the subcortical white matter becomes isointense with grey matter. This loss of grey/white matter differentiation obscures structural detail and makes identification of subtle abnormalities such as polymicrogyria difficult to detect within the first 6 months of life. The white matter shows maturity later, between 6 months and 2 years, on T2-W images and at this stage $\mathrm{T} 1-\mathrm{W}$ images are essential to evaluate structural abnormalities [4].

To overcome the difficulty of increased brain water in those under 2 years of age, we use a dual-echo short-tau inversion recovery (STIR) sequence that has improved contrast resolution in this age group (Fig. 1) [5]. Typical values for a dual-echo STIR sequence are TE $30 / 128 \mathrm{~ms}$, TR 5,400 $\mathrm{ms}$ and TI $130 \mathrm{~ms}$. We prefer this sequence to the more conventional $\mathrm{T} 2-\mathrm{W}$ fast (or turbo) spin-echo sequences, for reasons of increased lesion conspicuity. However,
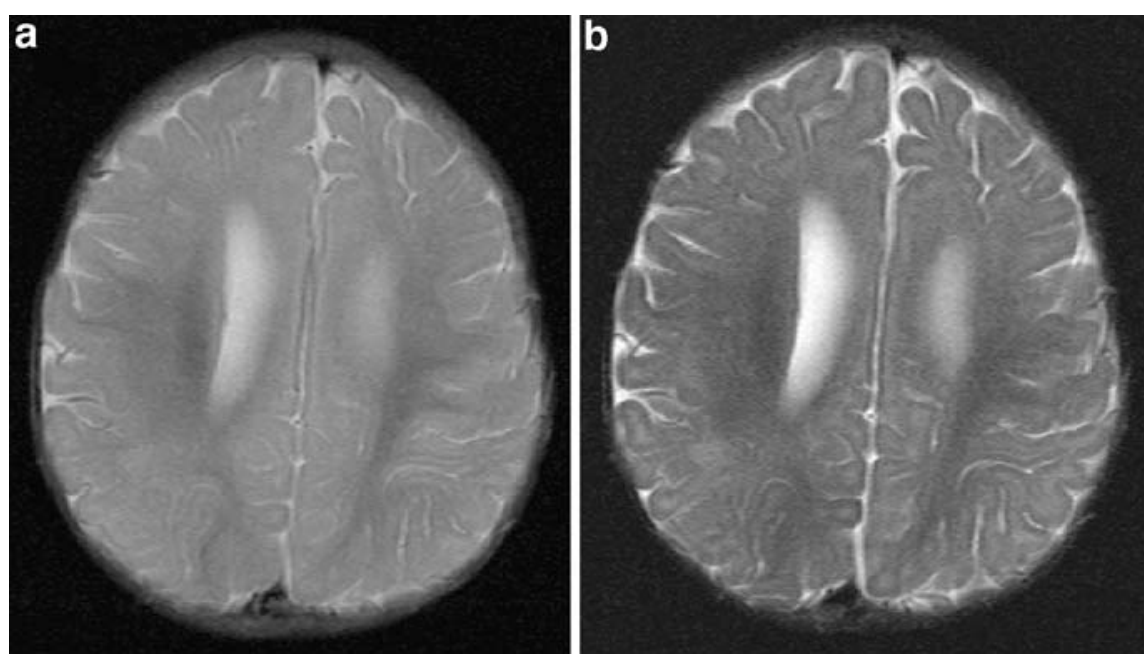

Fig. 1 Axial images acquired through the bodies of the lateral ventricles of a 6-month-old child. First (a) and second (b) echo (TE $30 / 120 \mathrm{~ms}$, TR 5,500 ms, TI $130 \mathrm{~ms}$ ) of a dual-echo short-tau sequence shows the increased contrast between grey and white matter on the second echo compared to (c) the T2-W (TE $90 \mathrm{~ms}$, TR $3,500 \mathrm{~ms}$ ) sequence that would be used in those over 2 years of age. The slice thickness $(5 \mathrm{~mm})$ and matrix size $(512 \times 192)$ were the same for both sequences 
we recognize that similar tissue contrast may be achieved if such fast spin-echo sequences are modified using increased TR and TE to compensate for the increased water content in this age group. The additional brain water is difficult to suppress and we do not find the fluid-attenuated inversion recovery (FLAIR) technique useful in this age group as lesion conspicuity is poor.

Diffusion measurements in the brain of newborns and infants show that there is more movement of water (high apparent diffusion coefficient, ADC) with less directionality (low anisotropy) within the immature and unmyelinated brain than in the mature and myelinated adult brain [6, 7]. The most dramatic decrease in ADC values occurs within the first few months of life and the greatest changes are seen in the frontal and parietal white matter [8]. Failure to appreciate the normal changes with age of the ADC will result in the erroneous reporting of white matter abnormality particularly in the neonatal period.

\section{Factors influencing sequence choice}

For any imaging protocol, it is the specific combination of sequences that determines the diagnostic efficacy of the examination. It is clear that imaging protocols vary between institutions. This is largely because rapid advances in imaging technology and variations between manufacturers, applied in the context of investigating relatively rare disorders, precludes an effective evidence-based approach to sequence choice.

The main advantages that MRI offers over alternative imaging modalities is the ability to demonstrate different tissue contrasts (principally T1-W, T2-W and spin density, but also flow and diffusion) in multiple imaging planes (principally sagittal, coronal and axial). The disadvantage of MRI is the artefacts that it generates in almost every image.

The choice of sequence combination should reflect the multicontrast and multiplanar capabilities of MRI. We have found that applying the generic principle of combining T2$\mathrm{W}$ images in two planes, supported by $\mathrm{T} 1-\mathrm{W}$ imaging in two planes, as the basis of our imaging protocols, serves to optimize the benefits of MRI whilst minimizing the impact of artefacts.

\section{Standard MRI studies}

Brain

Our standard brain sequence ( $>2$ years of age) is composed of an axial T2-W, coronal FLAIR, and coronal and sagittal
T1-W images. Sagittal T1-W images allow the assessment of the midline structures, particularly the corpus callosum and cerebellum, which are frequently abnormal in congenital abnormalities. Coronal T1-W images are valuable in the diagnosis of abnormalities of the anterior visual pathway, schizencephaly and holoprosencephaly. Axial T2-W and coronal FLAIR images provide T2-W images in two different planes and have been shown to be complementary in children [9]. DWI is acquired in all children unless artefacts from, for example, dental braces or a ventriculoperitoneal shunt, preclude it, and an ADC is calculated using automated computer software and provided for reporting.

In those under 2 years of age, the T2-W sequence is replaced by a dual-echo axial STIR sequence (see above). In some cases, a $\mathrm{T} 2 *-\mathrm{W}$ gradient-echo (GE) sequence ("susceptibility-weighted" sequence), sensitive to changes in local field inhomogeneity caused by the breakdown products of haemoglobin, is added. The sequence is particularly useful in trauma and vascular malformations such as multiple cavernomas.

Spine

MRI is the modality of choice for imaging the intraspinal components of the paediatric spine. Prior to ossification of the posterior elements of the spinal column, US has been shown to be a valuable screening tool; however, infants with an abnormal sonogram or who have a neurological abnormality in the context of a normal sonogram, still require MR imaging [10].

Our standard spine imaging includes sagittal, fast spinecho T1- and T2-W sequences (3-mm-thick slices). Both axial T1-W and T2-W images are acquired through any abnormality. Unlike most adult spine imaging protocols, groups of axial images through disc levels are not applied because degenerative disc disease is rare. Children with scoliosis and/or suspected spinal dysraphism routinely have axial T1-W images through the conus and filum terminale to detect lipomas of the filum terminale that may not be visible on sagittal imaging.

\section{Contrast medium}

The use of contrast medium is a personal choice and in the majority of units is requested on a case-by-case basis. At GOSH the radiographers or sedation nurses administer MR contrast medium according to guidelines set up by the neuroradiologists (Table 1). In the event of an unexpected finding the radiographers are encouraged to make a decision on contrast administration without discussion with a radiologist. 
Table 1 Indications for contrast medium administration

Various indications

Acute inflammation

Acute disseminated encephalomyelitis

(ADEM)

Optic neuritis

Acute infection

Abscess

Cerebritis

Discitis

Empyema

Encephalitis

Meningitis

Transverse myelitis

Neurocutaneous

disorders

Tumours

White matter

disorders $^{\mathrm{a}}$

Vascular anomalies

Cavernomas

Developmental venous anomalies

Vascular disorders Intraparenchymal haemorrhage

Sturge-Weber syndrome

Vasculitis

${ }^{\text {a }}$ At presentation and at follow-up if necessary

\section{MRI protocols for specific areas}

The strategies applied to the brain are also applied to small parts such as the orbits and pituitary gland. The orbit is scanned using a STIR sequence to benefit from the fat saturation properties of the sequence and improve the conspicuity of lesions within the orbit. The slice thickness is reduced to $3 \mathrm{~mm}$ and, to increase the signal-to-noise, the matrix size is reduced, thereby slightly reducing the inplane resolution.

A heavily T2-W 3-D volume sequence of the petrous temporal bones is used to image the membranous labyrinth and is reconstructed in the coronal plane of the petrous temporal bone, and the axial and sagittal plane of the internal auditory meati (IAM). The sagittal plane is used to view the vestibulocochlear nerve in cross section. A constructive interference steady state sequence (CISS), available on Siemens scanners, is acquired and reconstructed at a slice thickness of $0.7 \mathrm{~mm}$. The GE equivalent is the FIESTA sequence and the Philips equivalent is DRIVE.

\section{MRI protocols for specific neurological or neurosurgical presentations}

The majority of children undergoing an MRI brain scan will have epilepsy, stroke or a brain tumour and are often referred to specialists with an interest in these areas.

Tumours of the brain and spine

\section{Brain}

Imaging of brain tumours is performed using our MRI brain with contrast enhancement protocol. We add contrastenhanced T1-W imaging of the spine to establish the presence of any distant metastases in accordance with the United Kingdom Children's Cancer Study Group (UKCCSG) guidelines for the imaging of brain tumours [11]. One of the commonest reasons for repeat scanning of
Table 2 Proposed protocols for surveillance imaging in children with ependymoma, medulloblastoma and pilocytic astrocytoma

\footnotetext{
${ }^{a}$ All imaging studies in children with medulloblastoma should include the entire neuroaxis.

${ }^{\mathrm{b}}$ The surveillance imaging protocol following complete resection should be applied to children who have radiotherapy with the postoperative scan omitted
}

\begin{tabular}{|c|c|c|c|c|c|}
\hline \multirow[t]{3}{*}{ Tumour } & \multirow{3}{*}{$\begin{array}{l}\text { Timing of } \\
\text { postoperative } \\
\text { imaging }\end{array}$} & \multicolumn{4}{|c|}{ Frequency of study } \\
\hline & & \multicolumn{2}{|c|}{$\begin{array}{l}\text { Macroscopically complete } \\
\text { excision }\end{array}$} & \multicolumn{2}{|c|}{ Incomplete excision } \\
\hline & & $\begin{array}{l}\text { Cranial } \\
\text { study }\end{array}$ & $\begin{array}{l}\text { Spinal } \\
\text { study }\end{array}$ & $\begin{array}{l}\text { Cranial } \\
\text { study }\end{array}$ & $\begin{array}{l}\text { Spinal } \\
\text { study }\end{array}$ \\
\hline \multirow[t]{3}{*}{ Ependymoma } & $24-48 \mathrm{~h}$ & First & None & First & None \\
\hline & 1st year & 3-6 months & 6 months & 3 months & 3-6 months \\
\hline & $2 \mathrm{nd}-5$ th years & 6 months & 6-12 months & 6 months & 6-12 months \\
\hline \multirow[t]{3}{*}{ Medulloblastoma $^{\mathrm{a}}$} & $24-48 \mathrm{~h}$ & First & None & First & None \\
\hline & 1st year & 3-4 months & 3-4 months & 3-4 months & 3-4 months \\
\hline & 2nd-6th year & $6-8$ months & 6-8 months & 6-8 months & 6-8 months \\
\hline \multirow{6}{*}{$\begin{array}{l}\text { Cerebellar low-grade } \\
\text { astrocytoma }^{\mathrm{b}}\end{array}$} & $24-48 \mathrm{~h}$ & First & None & First & None \\
\hline & 1st year & 6 months & None & 6 months & None \\
\hline & 2 nd year & At 24 months & None & 6 months & None \\
\hline & 3rd year & At 3.5 years & None & 6 months & None \\
\hline & $4-5$ th year & At 5 years & None & 1 year & None \\
\hline & 6th year onwards & None & None & 2 years & None \\
\hline
\end{tabular}


a child with a brain tumour is the acquisition of contrastenhanced imaging of the spine in a child who has had only a brain scan at the referring centre.

The purpose of preoperative imaging of brain tumours is to assess tumour location and type, establish whether there are single or multiple lesions, define its relationship with vital structures and look for any complications such as hydrocephalus. Contrast-enhanced T1-W volumetric acquisitions are also acquired for image-guided surgery.

Imaging of the spine is performed in all children with intracranial tumours (not only posterior fossa tumours) and although this requires a change of coil and an additional sequence, we find it beneficial as tumour histology is often not certain at the time of the initial imaging. Some tumours unexpectedly metastasize to the spine and assessment of the postoperative spine is made more difficult in the presence of blood products.

Postoperative MRI is acquired using our MRI brain with contrast enhancement protocol to assess the degree of tumour clearance. We aim to do this within $72 \mathrm{~h}$ of surgery to reduce enhancement due to postoperative changes that can complicate the assessment of tumour clearance, in accordance with guidelines proposed by the UKCCSG [11]. If preoperative spinal imaging has not been acquired, the spine is imaged with both unenhanced and contrastenhanced $\mathrm{T} 1-\mathrm{W}$ imaging to enable differentiation between haematoma and tumour metastases.

Surveillance imaging (SI) during adjuvant treatment and follow-up is a major part of the management of childhood tumours and scanning frequency has important implications to anaesthetic and radiology service provision. In children with ependymoma and medulloblastoma, studies at GOSH have shown that SI reveals a substantial number of asymptomatic recurrences and survival appears to be improved in these patients compared with those patients in whom the recurrence is identified by symptoms $[12,13]$. Benefits of SI following incomplete resection of posterior fossa low-grade pilocytic astrocytoma have also been shown $[14,15]$. SI schedules have been written based on this work (Table 2).

\section{Spine}

Imaging of spinal tumours is performed using our MRI spine with contrast enhancement protocol (Table 3) with dedicated axial imaging through the tumour. We add a standard brain scan with contrast enhancement to the initial or immediate postoperative scan to stage the tumour. If the brain scan is normal, further brain imaging is not acquired as part of SI and acquired only when clinically indicated (Table 4).

\section{Epilepsy}

UK guidelines for the imaging of epilepsy include children who have either multiple or focal seizures (NICE guidelines) [16]. In children with epilepsy we are aiming to detect focal cortical abnormalities; the majority are extratemporal and in a smaller proportion they are mesial temporal in origin. The majority of cortical abnormalities are easily diagnosed using conventional brain imaging and our standard brain protocol is used to investigate these children. The acquisition of a coronal $\mathrm{T} 2-\mathrm{W}$ sequence (either STIR, T2-W or FLAIR) allows the visualization of the mesial temporal lobe, and is a sequence often not acquired in referring centres.

Table 3 Protocols for specific areas

\begin{tabular}{|c|c|}
\hline Specific areas & Protocols \\
\hline Orbits (3-mm slices) & $\begin{array}{l}\text { Coronal and axial dual-echo STIR } \\
\text { Coronal and axial T1-W spin-echo }\end{array}$ \\
\hline Orbits with contrast enhancement (3-mm slices) & $\begin{array}{l}\text { Coronal dual-echo STIR } \\
\text { Coronal and axial T1-W spin-echo } \\
\text { Contrast-enhanced coronal and axial T1-W images with fat saturation }\end{array}$ \\
\hline Pituitary (3-mm slices) & $\begin{array}{l}\text { Sagittal and coronal T1-W spin-echo } \\
\text { Coronal T2-W spin-echo }\end{array}$ \\
\hline Pituitary with contrast enhancement (3-mm slices) & $\begin{array}{l}\text { Pituitary protocol } \\
\text { Contrast-enhanced coronal and sagittal T1-W spin-echo }\end{array}$ \\
\hline Internal auditory meati & $\begin{array}{l}\text { 3-D volume axial CISS } \\
\text { Brain MRI }\end{array}$ \\
\hline Face and neck MRI & $\begin{array}{l}\text { Coronal and axial dual-echo STIR } \\
\text { Coronal and axial T1-W spin-echo } \\
\text { Fat-saturated contrast-enhanced coronal and axial T1-W spin-echo }\end{array}$ \\
\hline Midline facial lesions & $\begin{array}{l}\text { Axial dual-echo STIR from floor of anterior cranial fossa to hard palate } \\
\text { Sagittal T1-W and T2-W spin-echo (3-mm slices) } \\
\text { Coronal T1-W spin-echo from nose to brainstem }\end{array}$ \\
\hline
\end{tabular}


There are a group of children with intractable epilepsy, i.e. seizures not controlled by three drugs, who are considered for epilepsy surgery. In this subset of children a more rigorous epilepsy protocol is carried out that includes a 3-D volume T1-W acquisition and hippocampal T2 relaxometry (Table 5). To identify mesial temporal abnormalities the coronal studies are planned on a parasagittal scout image to lie perpendicular to the hippocampi. The 3-D, T1-W gradient-echo dataset is acquired isometrically in the sagittal plane (this saves time) and reconstructed in all three planes with the axial and coronal images tilted to follow the plane of the hippocampus. More recently a 3-D FLAIR sequence reconstructed along the same lines has been added. A sequence is acquired that measures the true $\mathrm{T} 2$ values of the hippocampi (T2 relaxometry), which is only of value in suspected mesial temporal sclerosis (MTS). It has been shown that $\mathrm{T} 2$ values are more sensitive to the presence of MTS than visual inspection and T2 relaxometry is of particular value in bilateral disease [17]. Normal values will vary between scanners, so normative data should be acquired. The radiographers carry out the postprocessing of the 3-D dataset and the $\mathrm{T} 2$ relaxometry.
Non-traumatic intraparenchymal haemorrhage

The aim of imaging children with intraparenchymal haemorrhage (IPH) is to detect the underlying cause and we advocate the use of CT angiography as a matter of urgency in those children who have a depressed level of consciousness or whose clinical condition is deteriorating. MRI is used to investigate clinically stable children, and we perform brain MRI with contrast enhancement and a 3-D, time-of-flight (TOF) intracranial MR angiography (MRA). In our experience nearly two-thirds of children presenting with nontraumatic IPH have an underlying cause diagnosed by MRI and MRA, with aetiologies including tumour, arteriovenous malformation (AVM), venous sinus thrombosis and cavernous angioma [18]. An attempt is made to perform MRI within $72 \mathrm{~h}$ of the ictus to overcome the problem of the visibility of blood products (T1 shortening due to methaemoglobin) on 3-D TOF sequences that may obscure the underlying pathology. The value of intravenous contrast agents in identifying underlying vascular causes has not been proven. We have a largely anecdotal view that it is helpful in identifying small AVMs and venous anomalies.

Table 4 Standard MRI brain and spine protocols

\begin{tabular}{|c|c|}
\hline Types of brain and spine MRI & Protocols \\
\hline MRI brain (under 2 years old) & $\begin{array}{l}\text { Axial and coronal dual echo STIR } \\
\text { Coronal and sagittal T1-W spin-echo } \\
\text { DWI in three planes and calculated ADC map } \\
\text { Axial "susceptibility-weighted" GE sequence }\end{array}$ \\
\hline MRI brain (over 2 years old) & $\begin{array}{l}\text { Axial T2-W fast spin-echo } \\
\text { Coronal FLAIR } \\
\text { Coronal and sagittal T1-W spin-echo } \\
\text { DWI in three planes and calculated ADC map } \\
\text { Axial "susceptibility-weighted" GE sequence }\end{array}$ \\
\hline $\begin{array}{l}\text { MRI brain with contrast enhancement } \\
\text { (under } 2 \text { years old) }\end{array}$ & $\begin{array}{l}\text { Axial and coronal dual-echo STIR } \\
\text { Coronal T1-W spin-echo } \\
\text { DWI in three planes and calculated ADC map } \\
\text { Contrast-enhanced axial, coronal and sagittal T1-W spin-echo with magnetization transfer } \\
\text { Axial "susceptibility-weighted" GE sequence }\end{array}$ \\
\hline $\begin{array}{l}\text { MRI brain with contrast enhancement } \\
\text { (over } 2 \text { years old) }\end{array}$ & $\begin{array}{l}\text { Axial T2-W fast spin-echo } \\
\text { Coronal FLAIR } \\
\text { Coronal T1-W spin-echo } \\
\text { DWI in three planes and calculated ADC map } \\
\text { Contrast-enhanced axial, coronal and sagittal T1-W spin-echo with magnetization transfer } \\
\text { Axial "susceptibility-weighted" GE sequence }\end{array}$ \\
\hline MRI spine & $\begin{array}{l}\text { Sagittal T1-W and T2-W fast spin-echo } \\
\text { Axial T1-W and T2-W fast spin-echo through target area and conus } \\
\text { (Coronal T1-W spin-echo for scoliosis, if patient compliant) }\end{array}$ \\
\hline MRI spine with contrast enhancement & $\begin{array}{l}\text { Sagittal T1-W and T2-W fast spin-echo } \\
\text { Contrast-enhanced sagittal T1-W fast spin-echo } \\
\text { Axial T1-W spin-echo through target area }\end{array}$ \\
\hline
\end{tabular}

${ }^{\mathrm{a}}$ Optional sequence 
Table 5 Protocols for particular clinical indications

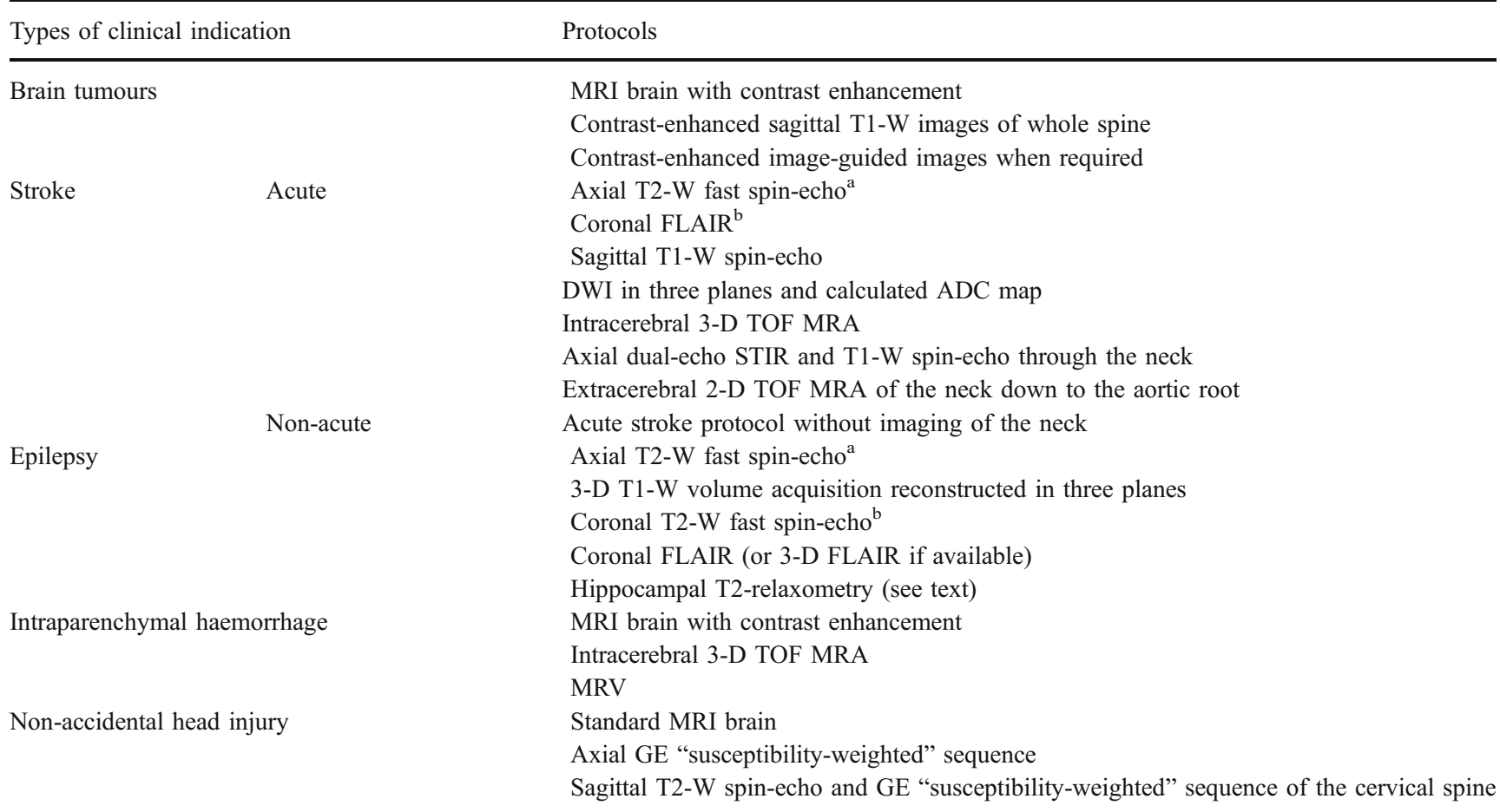

$M P R A G E$ magnetization prepared rapid acquisition gradient echo, $M R V$ magnetic resonance venography

${ }^{\text {a }}$ Replaced by dual echo STIR sequence in the under 2 age group

${ }^{\mathrm{b}}$ Replaced by a dual echo STIR coronal in the under 2 year age group.

\section{Stroke}

The aims of conventional MRI are not only to detect the infarct, but also to provide information to establish the cause of the stroke and to exclude other causes (such as tumour or infection). The majority of children with stroke have a combination of risk factors including sickle cell disease, congenital heart disease, anaemia, prothrombotic disorders and infections such as varicella-zoster [19]. The rationale for the inclusion of intracranial MRA in all children with stroke is that cerebral arteriopathy is found in up to $80 \%$ of these children and most commonly affects focal areas of large intracranial arteries [19]. Specific entities such as moyamoya disease may also be diagnosed. The commonest abnormality identified is occlusion or stenosis, of unknown aetiology, affecting the terminal internal carotid artery (ICA) or proximal middle cerebral artery (MCA).

Extracranial MRA and, either a dual-echo STIR or fatsaturated axial T1-W imaging, through the neck can detect arterial dissection, particularly in children with posterior circulation infarcts [20]. The inversion time of the STIR sequence is selected to suppress the fat within the neck. The fat saturation provided by both these sequences improves the conspicuity of the haematoma within the extracranial vessel wall.

A 3-D TOF MRA sequence is used for the imaging of the intracranial vessels and a 2-D TOF MRA sequence for the extracranial vessels. The TOF scan times are shorter than phase-contrast (PC) MRA and there is lack of dependence on the choice of correct velocity encoding with obvious advantages when scanning ill children. Intracranial 3-D TOF MRA is also included in the investigation of children with IPH, although its sensitivity to $\mathrm{T} 1$ shortening may obscure the underlying abnormality (see above).

As CT is often the first-line investigation in children with stroke, the potential of DWI to detect hyperacute cerebral infarction prior to changes on T2-W MRI is not realized. However, inpatients (e.g. cardiac patients, patients with recent-onset stroke) can be imaged early, and in these children DWI can be used to detect infarcts of different ages.

Non-accidental head injury

Although CT is the primary imaging tool in the evaluation of nonaccidental head injury (NAHI), we perform MRI in children with an abnormal CT or neurological symptoms 
and signs on day 3-4 in accordance with the protocol proposed by Jaspan et al. [21]. The rationale for performing MRI is that it is more sensitive than CT to both hypoxicischaemic injury, particularly when DWI is used, and subdural haematomas adjacent to the calvarium or in the posterior fossa. MRI is repeated at $2-3$ months in those children with parenchymal brain injury or a persistent neurological abnormality.

Children with suspected NAHI are imaged using our standard brain protocol. In addition axial GE (susceptibility-weighted) sequences are acquired, as they are more sensitive to blood products than standard sequences. Sagittal T2-W and GE images are acquired of the cervical cord, including the brainstem, particularly to identify shear injuries within the brainstem that have been proposed as a mechanism of hypoxic-ischaemic injury in children who have been shaken as the mechanism of injury [22, 23]. The aim of the sagittal T2-W sequence of the cervical cord is to look for any evidence of trauma as a result of violent shaking in an attempt to establish the true, albeit rare, incidence of pathology in the upper cervical spine [21].

\section{Neonatal imaging}

Conventional imaging can detect patterns of regional brain injury in the neonatal period that can help time the injury, determine underlying mechanisms and ultimately provide some prognostic information. MRI is useful in detecting hypoxic-ischaemic injury, and germinal matrix and intraventricular haemorrhage, and can be useful to distinguish other pathologies that may mimic hypoxic-ischaemic encephalopathy in the neonatal period such as venous infarction, metabolic disease, infection, and congenital developmental abnormalities.

Our neonatal imaging protocol is the same as the under2 -year brain protocol. Achieving high signal-to-noise is of utmost importance given the small size of the infant head and is improved by decreasing the slice thickness to $4 \mathrm{~mm}$ whilst decreasing the matrix size from $512 \times 512$ to $256 \times$ 256 in a field-of-view of $180 \mathrm{~mm}$. Although specific neonatal head coils have been developed, most nonpaediatric centres are unlikely to have access to them and improved results can be obtained by using an adult knee coil.

\section{Developmental delay}

Developmental delay, without other clinical features such as epilepsy or dysmorphic features, is not considered an indication for an MRI scan at our institution. This decision was made in conjunction with our neurologists as it was recognized that there is an extremely low yield of clinically relevant abnormalities seen in patients with developmental delay alone.

\section{The future}

The future of paediatric neuroimaging lies in the incorporation of research techniques, such as perfusion imaging and diffusion tensor imaging, into standard imaging protocols once they have been demonstrated to be of value within the clinical arena. The use of higher field strength magnets will have the advantage of increasing signal-to-noise and reducing scanning time, which will be of benefit to the child who is both small and liable to move.

\section{References}

1. Sury MR, Hatch DJ, Deeley T et al (1999) Development of a nurse-led sedation service for paediatric magnetic resonance imaging. Lancet 56:388-391

2. Sury MR, Harker H, Begent J et al (2005) The management of infants and children for painless imaging. Clin Radiol 60:731-741

3. Barkovich AJ (2000) Concepts of myelin and myelination in neuroradiology. AJNR 21:1099-1109

4. Barkovich AJ (2005) Pediatric neuroimaging, 4th edn. Lippincott Williams \& Wilkins, Philadelphia, chapter 1

5. Finn JP, Connelly A, Atkinson D (1989) A modified inversion recovery sequence for routine high-contrast brain imaging (abstract). Abstract book of the Society of Magnetic Resonance Eighth Annual Meeting and Exhibition, vol 2, p 722

6. Morris MC, Zimmerman RA, Bilaniul LT et al (1999) Changes in brain water diffusion in childhood. Neuroradiology 41:929-934

7. Mukherjee P, Miller JH, Shimony JS et al (2001) Normal brain maturation during childhood; developmental trends characterized with diffusion tensor imaging. Radiology 221:349-358

8. Forbes KP, Pipe JG, Bird CR (2002) Changes in brain water during the first year of life. Radiology 222:405-409

9. Barkovich AJ (2005) Pediatric neuroimaging, 4th edn. Lippincott Williams \& Wilkins, Philadelphia, chapter 2

10. Hughes JA, De Bruyn R, Patel K et al (2003) Three-dimensional sonographic evaluation of the infant spine: preliminary findings. J Clin Ultrasound 31:9-20

11. Griffiths PD (1999) A protocol for imaging paediatric brain tumours. United Kingdom Children's Cancer Study Group and Societe Francaise d'Oncologie Pediatrique Panelists. Clin Radiol $54: 558-562$

12. Good CD, Wade AM, Hayward RD et al (2001) Surveillance neuroimaging in childhood intracranial ependymoma: how effective, how often, and for how long? J Neurosurg 94:27-32

13. Saunders DE, Hayward RD, Phipps KP et al (2003) Surveillance neuroimaging of intracranial medulloblastoma in children: how effective, how often, and for how long? J Neurosurg 99:280-286

14. Gunny RS, Hayward RD, Phipps KP et al (2005) Spontaneous regression of residual low-grade cerebellar pilocytic astrocytomas in children. Pediatr Radiol 35:1086-1091

15. Saunders DE, Phipps KP, Wade A et al (2005) Surveillance imaging strategies following surgery and/or radiotherapy for childhood cerebellar low-grade astrocytoma. J Neurosurg 102:172-178

16. National Institute for Health and Clinical Excellence (2007) Epilepsy.The epilepsies: the diagnosis and management of the epilepsies in adults and children in primary and secondary care. National Institute for Health and Clinical Excellence, London. http:// 
www.nice.org.uk/guidance/CG20/niceguidelines/pdf/English. Accessed 27 March 2007

17. Scott RC, Gadian DG, Cross JH et al (2001) Quantitative magnetic resonance characterization of mesial temporal sclerosis in childhood. Neurology 56:1659-1665

18. Liu AC, Ganesan V, Hayward R et al (2006) Is there a role for magnetic resonance imaging in the diagnosis of non-traumatic intraparenchymal haemorrhage in children? Pediatr Radiol 36:940-946

19. Ganesan V, Prengler M, McShane MA et al (2003) Investigation of risk factors in children with arterial ischaemic stroke. Ann Neurol 53:167-173
20. Ganesan V, Chong WK, Cox TC et al (2002) Posterior circulation stroke in childhood: risk factors and recurrence. Neurology 59:1552-1556

21. Jaspan T, Griffiths PD, McConachie NS et al (2003) Neuroimaging for non-accidental head injury in childhood: a proposed protocol. Clin Radiol 58:44-53

22. Geddes JF, Hackshaw AK, Vowels GH et al (2001) Neuropathology of inflicted head injury in children. I. Patterns of brain damage. Brain 124:1290-1298

23. Geddes JF, Vowels GH, Hackshaw AK et al (2001) Neuropathology of inflicted brain injury in children. II. Microscopic brain injury in infants. Brain 124:1299-1306 